\title{
Responsabilidad social, necesidades psicológicas básicas, motivación intrínseca y metas de amistad en educación física

\author{
Social responsibility, basic psychological needs, intrinsic motivation, and friendship goals in physical \\ education \\ José Ignacio Menéndez Santurio, Javier Fernández-Río \\ Universidad de Oviedo (España)
}

\begin{abstract}
Resumen. El objetivo de este estudio fue analizar la capacidad de predicción de la responsabilidad social, las necesidades psicológicas básicas y la motivación sobre la meta de aproximación a la amistad a partir de la Teoría de la Autodeterminación. Una muestra de 402 estudiantes (209 mujeres y 193 hombres; $M=14.20 ; D T=1.52$ ) de entre 12 y 18 años, procedentes de tres centros de educación secundaria del norte de España cumplimentaron un cuestionario que incluía la subescala de responsabilidad social del Cuestionario de la Responsabilidad Personal y Social (PSQR), la Escala de Necesidades Psicológicas Básicas en Educación Física (BPN-PE), la subescala de motivación intrínseca de la Escala del Locus Percibido de Casualidad (PLOC) y la subescala de aproximación-amistad del Cuestionario de Metas de Amistad (FGQ-PE). Los resultados del análisis de regresión lineal múltiple reflejaron que la responsabilidad social, las necesidades psicológicas básicas de relación y autonomía y la motivación intrínseca predecían significativamente la meta de aproximación-amistad. Estos resultados reflejan la importancia de crear contextos educativos en los que se potencie la responsabilidad social, las relaciones, la autonomía y la motivación intrínseca de los estudiantes con el objetivo de mejorar sus metas de amistad.

Palabras clave. Autodeterminación, modelo de Vallerand, educación física, mediadores psicológicos, motivación.
\end{abstract}

\begin{abstract}
The goal of the present study was to assess social responsibility, basic psychological needs, and motivation towards friendship-approach goals within the Self-Determination Theory. A total of 404 students from three secondary schools of northern Spain (209 females and 193 males; $M=14.20$; $D T=1.52$ ), aged from 12 to 18 years, completed a questionnaire that included the social responsibility subscale of the Personal and Social Responsibility Questionnaire (PSQR), the Basic Psychological Needs in Physical Education Scale (BPN-PE), the subscale of intrinsic motivation of the Perceived Locus of Causality (PLOC), and the subscale of friendship-approach of the Friendship Goals Questionnaire in Physical Education (FGQ-PE). Results of multiple linear regression analysis showed that social responsibility, relatedness and autonomy, and intrinsic motivation significantly predicted friendship-approach goals. These results suggest the importance of creating educational contexts that can foster social responsibility, relatedness, autonomy, as well as students' intrinsic motivation, with the aim to improve their friendship goals.
\end{abstract}

Keywords. Self-determination, Vallerand's model, physical education, psychological mediators, motivation.

\section{Introducción}

La práctica regular de actividad física es uno de los elementos fundamentales para adquirir estilos de vida saludables y un adecuado bienestar psicológico (Jiménez, Martínez, Miró \& Sánchez, 2008). En ese sentido, la educación física proporciona uno de los contextos más propicios para favorecer la calidad de vida de niños y adolescentes (Standage \& Gillison, 2007). Además, otorga un ambiente óptimo donde los estudiantes pueden potenciar el desarrollo de habilidades sociales, fomentar vínculos de amistad con sus pares y resolver problemas de forma pacífica (Monzonís \& Capllonch, 2014).

La responsabilidad es definida como «la obligación moral que una persona tiene sobre uno mismo y sobre los demás» (Menéndez \& Fernández-Río, 2016a, p. 247). Existen dos tipos fundamentales de responsabilidad: de carácter personal y de índole social. A cada una de ellas están asociados diversos componentes y/o valores. Por ejemplo, a la responsabilidad personal le corresponden valores como el respeto a los derechos y opiniones de los demás, la sensibilidad social y la empatía, mientras que a la responsabilidad social le corresponden valores como el esfuerzo y la autonomía (Hellison, 2011). La responsabilidad ha sido ampliamente estudiada, especialmente dentro de uno de los marcos de referencia más significativos: el Modelo de la Responsabilidad Personal y Social (MRPS; Hellison, 2011). Los múltiples trabajos que se han realizado sobre la responsabilidad dentro del ámbito de la educación física (Caballero, Delgado \& Escartí, 2013; Hemphill, Templin \& Wright, 2013; Menéndez \& Fernández-Río, 2016b; Severinsen, 2014; SánchezAlcaraz, Gómez-Mármol, Valero \& De la Cruz, 2013) han revelado la importancia de esta variable para fomentar el llamado desarrollo positivo (Seligman \& Csikszentmihalyi, 2000). Mientras que la responsabilidad personal tiene un gran impacto sobre componentes como la automotivación o la disciplina, el calado de la responsabilidad social recae, especialmente, en la relación que tiene esta con conductas sociales

Fecha recepción: 25-09-16. Fecha de aceptación: 22-01-17 José Ignacio Menéndez Santurio U0194643@uniovi.es adaptativas, con el desarrollo de la amistad, con la relación entre el alumnado o con el juego limpio (Fernández-Río, Méndez-Giménez, Cecchini-Estrada \& González-Mesa, 2012; Menéndez \& FernándezRío, 2016a).

La Teoría de la Autodeterminación (TAD; Deci \& Ryan, 2002) se ha establecido como uno de los marcos de referencia más significativos para el estudio de los procesos motivacionales. Esta señala que las motivaciones de un individuo se ven reflejadas a lo largo de un continuo de autodeterminación conformado por diferentes tipos de regulaciones motivacionales: motivación intrínseca, motivación extrínseca y desmotivación. La motivación intrínseca hace referencia al hecho de realizar cualquier actividad por propio placer e interés. Es un tipo de motivación de carácter totalmente hedonista. Por su parte, la motivación extrínseca hace referenciaa aquellas conductas que están influenciadas por factores externos. Finalmente, la desmotivación se caracteriza por la ausencia de motivación interna o externa (Deci \& Ryan, 1985). De todas ellas, la motivación intrínseca es la de mayor importancia, pues a la luz de los estudios que se han llevado a cabo tanto con jóvenes deportistas como con estudiantes de educación física han revelado que este tipo de motivación está relacionada con otras conductas adaptativas como la satisfacción con la vida (Méndez-Giménez, Cecchini-Estrada, Fernández-Río, Méndez-Alonso \& Prieto-Saborit, 2017), la autoestima y la diversión (Moreno \& Vera, 2011), el motivo social de práctica física (Moreno, Águila \& Borges, 2011), los comportamientos prosociales (Sánchez, Leo, Sánchez, Gómez \& García, 2011), el aprendizaje cooperativo (Fernández-Río, Cecchini-Estrada \& Méndez-Giménez, 2014), la intención de práctica futura (Franco, Coterón, Gómez, Brito \& Martínez, 2017) o la autoconfianza (Sari, Ekici, Soyen \& Eskiler, 2015), entre otras.

Asimismo, la TAD establece la existencia de tres necesidades psicológicas básicas (competencia, autonomía y relación) que están estrechamente vinculadas con el contexto social del individuo y que pueden afectar a los diferentes tipos de motivación existentes (Deci \& Ryan, 2002). La competencia es la percepción que los sujetos tienen sobre su propia capacidad para realizar una tarea en un contexto determinado (Deci \& Ryan, 2002). La relación se entiende como la sensación de 
pertenencia a un grupo u entorno social concreto (Vlachopoulos \& Michailidou, 2006). Finalmente, la autonomía hace referencia al grado en que los sujetos sienten que tienen poder para dirigir y controlar sus propias conductas (Niemec \& Ryan, 2009). Las investigaciones sobre este postulado de la TAD han revelado que la satisfacción de estas tres necesidades psicológicas básicas se relaciona con mayores niveles de autodeterminación y motivación intrínseca (Cox \& Williams, 2008; Ntoumanis, 2001, 2005; Standage, Duda \& Ntoumanis, 2003), con la predisposición positiva hacía la práctica de diversos contenidos en educación física (Sevil,Abós, Generelo, Aibar \& García González, 2016), el aprendizaje cooperativo (Méndez-Giménez, Cecchini-Estrada \& Fernández-Río, 2013a), el autoconcepto y el papel importante del alumnado (Méndez-Giménez, Cecchini-Estrada \& Fernández-Río, 2013b), el disfrute (Navarro-Patón, Rodríguez \& Eirín, 2016), la intención de práctica física en tiempo libre (Granero-Gallegos, BaenaExtremera, Sánchez-Fuentes \& Martínez-Molina, 2014), la percepción de apoyo a la autonomía del entrenador (Cantú-Berrueto, Castillo, López-Walle, Tristán \& Balaguer, 2016) o de forma negativa con el aburrimiento (García-González,Aibar, Sevil,Almolda \& Clemente, 2015; Méndez-Giménez, Cecchini-Estrada \& Fernández-Río, en prensa) y con las conductas desafiantes (Abós, Sevil, Sanz, Aibar \& GarcíaGonzález, 2016)

Por otro lado, uno de los marcos más relevantes para el estudio de la motivación es la Teoría de Metas de Logro (Nicholls, 1989). En los inicios, Nicholls (1989) propuso dos tipos de metas: las de implicación a la tarea y las de implicación al ego. Estas metas se establecieron con base a la definición que Elliot (1999) otorgó al concepto competencia. Posteriormente, Elliot \& McGregor (2001) añadieron a este marco dicotómico la dimensión valencia. Así pues, se podrían dar dos formas de metas: de aproximación, es decir, comportamientos que se aproximan hacia estímulos positivos, y de evitación, a saber, conductas que tienden a alejarse de los estímulos negativos (Elliot, Gable \& Mapes, 2006). Sin embargo, la Teoría de la Motivación Social (TMS, Allen, 2003) afirma que las metas de acción no proceden únicamente de la percepción que un individuo tiene sobre sus habilidades, sino que también proviene de la percepción que este tiene sobre aspectos de carácter social, como puede ser la afiliación, la cohesión de grupo, o el sentimiento de pertenencia a un grupo. Este enfoque también entiende que la motivación es un proceso de tipo psicológico, pero hace especial mención en que en contextos sociales como puede ser el físico-deportivo, el potenciador más significativo de la motivación es el afán de adquirir, facilitar y mantener lazos sociales con el resto de personas (Allen, 2003). Posteriormente, Elliot et al. (2006) añadieron la dimensión de valencia al marco de la variable amistad. Así pues, se pueden dar dos formas de metas: de aproximación, es decir, comportamientos que se representan por el esfuerzo por lograr la competencia social (e.g., fomentar las relaciones sociales con tus pares) y de evitación, a saber, conductas orientadas a evitar la incompetencia social (e.g., evitar conflictos en las relaciones sociales) (Elliot et al., 2006).

Con base en las aportaciones de la TAD, Vallerand (1997) planteó el Hierarchical Model of Intrinsic and Extrinsic Motivation, que fija una secuencia de cuatro etapas de índole causal: Factores sociales $\rightarrow$ Mediadores Psicológicos $\rightarrow$ Tipos de Motivación $\rightarrow$ Consecuencias. El Modelo Jerárquico de Vallerand (1997) contribuyó de forma significativa a la TAD. Vallerand fijóla existencia de tres niveles jerárquicos: situacional, contextual y global. El primero de ellos se refiere a la motivación que un individuo posee en un momento concreto. El segundo caso está relacionado con las motivaciones que ese sujeto tiene en un contexto particular (e.g., amigos, trabajo, familia, colegio, etc.). Finalmente, la motivación de tipo global se centra en explicar que a lo largo de las diversas situaciones que acontecen, una persona puede experimentar diversos tipos de motivación en contextos dispares. El autor clarificó que hay un estrecho vínculo entre los tres niveles, de forma que las motivaciones de un nivel pueden tener efectos en las otras dos. A pesar de que varios autores han estudiado esta secuencia con diferentes tipos de factores sociales y consecuencias (Cox \& Williams, 2008; Méndez-Giménez et al., 2013a, b, en prensa; Moreno, Cervelló, Montero, Vera \& García, 2012;
Ntoumanis, 2001; Standage et al., 2003), hasta la fecha ningún trabajo ha tenido en cuenta la responsabilidad social como factor social y la meta de aproximación-amistad como consecuencia en el Modelo Jerárquico de Vallerand (1997). Sin embargo, Elliot et al. (2006) apuntan que es fundamental que se analicen los dominios de tipo social para poder tener una visión sobre la motivación del alumnado adolescente de mayor transcendencia. Otras investigaciones realizadas en el ámbito de la motivación social han apoyado esta idea (Cecchini, González-Mesa, Méndez-Giménez \& Fernández-Río, 2011; González-Cutre, Sicilia \& Moreno, 2008).

Así pues, el objetivo principal de este estudio fue estudiar la capacidad de predicción de la responsabilidad social, las necesidades psicológicas básicas y la motivación intrínseca sobre la meta de aproximación-amistad. La hipótesis principal que se planteó fue la siguiente: la responsabilidad social, las necesidades psicológicas básicas de competencia, relación y autonomía, y la motivación intrínseca predecirán positiva y significativamente la meta de aproximación-amistad.

\section{Método}

\section{Participantes y diseño}

En el estudio participaron 402 estudiantes pertenecientes a tres institutos de educación secundaria del norte de España (12 grupos naturales). El nivel socioeconómico de los centros era de carácter medio. De ellos, 209 eran mujeres (52\%) y 193 hombres (48\%). Los estudiantes de la investigación eran de $1^{\circ}$ de la ESO (Educación Secundaria Obligatoria) $\mathrm{a} 1^{\circ}$ de bachillerato. Las edades de la muestra oscilaban entre los 12 y los 18 años $(M=14.20 ; D T=1.52)$. Se empleó una técnica de muestreo no probabilístico intencional por accesibilidad (Bisquerra, 2012). En la Tabla 1 se señala la distribución de la muestra en función del sexo y la edad. El diseño de la investigación fue ex post facto de carácter transversal y correlacional (Montero \& León, 2005).

Tabla 1.

\begin{tabular}{ccc}
\multicolumn{3}{l}{ Distribución de la muestra en función del sexo y la edad. } \\
\hline \multicolumn{2}{c}{ Edad (años) } & \multicolumn{3}{l}{ Sexo } \\
\hline & Hombres & Mujeres \\
\cline { 2 - 3 } 12 & 45 & 30 \\
13 & 35 & 25 \\
14 & 44 & 42 \\
15 & 56 & 47 \\
16 & 16 & 30 \\
17 & 12 & 17 \\
18 & 1 & 2 \\
\hline
\end{tabular}

\section{Instrumentos}

Responsabilidad social. Se utilizó la subescala de responsabilidad social de la versión española del Personal and Social Responsibility Questionnaire de Li, Wright, Rukavina \& Pickering (2008) validada por Escartí, Gutiérrez \& Pascual (2011). Esta subescala está compuesta por 7 ítems (e.g., «Respeto al profesor»). Las instrucciones iniciales del cuestionario dicen lo siguiente: «Lo normal es comportarse unas veces bien y otras mal. Nos interesa saber cómo te comportas normalmente durante la clase de Educación Física. No hay respuestas correctas o incorrectas. Por favor, responde a las siguientes preguntas con sinceridad y rodea con un círculo el número». La escala se puntúa con una escala Likert con 6 alternativas que oscilan desde (1) totalmente en desacuerdo a(6) totalmente de acuerdo. Se calculó el alpha de Cronbach, cuyo valor fue de 83 .

Necesidades Psicológicas básicas. Se empleó la versión española validada (Menéndez \& Fernández-Río, en prensa) del Basic Psychological Needs in Physical Education Scale (BPN-PE) de Vlachopoulos, Katartzi \& Kontou (2011). La introducción del cuestionario dice lo siguiente: «En general en Educación Física...». La escala está conformada por 12 ítems agrupados en 3 dimensiones de 4 ítems cada una de ellas: competencia (e.g., «Tengo éxito incluso en las clases que la mayoría de los compañeros considera difíciles»), relación (e.g., «Siento que soy un miembro valioso de un grupo de buenos amigos») y autonomía (e.g., «Siento como si las actividades que realizamos las hubiese escogido yo mismo»). Las respuestas están recogidas en una 
escala tipo Likert con 7 alternativas que oscilan de (1) totalmente en desacuerdo a (7) totalmente de acuerdo. El alpha deCronbach fue de.91 para la competencia, de .80 para la relación, de .86 para la autonomía y de .89 para la escala completa.

Motivación intrínseca. Se utilizó la subescala de motivación intrínseca del Perceived Locus of Causality (Goudas, Biddle \& Fox, 1994), validada al español por Moreno González-Cutre \& Chillón (2009). En la introducción del cuestionario dice lo siguiente: «Participo en esta clase de Educación Física...». La subescala está compuesta por 4 ítems (e.g., «Por la satisfacción que siento mientras aprendo nuevas habilidades/ técnicas»). La escala se puntúa con una escala Likert de 7 puntos que oscilan desde (1) totalmente en desacuerdo a (7) totalmente de acuerdo. El alpha de Cronbach fue de .88 .

Meta de aproximación a la amistad. Se empleó la subescala de aproximación-amistad de la versión española (Méndez-Giménez, Fernández-Río \& Cecchini-Estrada, 2014) del Relationship Goals Questionnaire-Friendship Version de Elliot et al. (2006). La raíz previa dice: «En mis clases de Educación Física trato de...». La subescala se compone de 4 ítems (e.g., «Profundizar en las relaciones con mis amigos»). Las respuestas se recogen en una escala tipo Likert que oscila de (1) totalmente en desacuerdo a (5) totalmente de acuerdo. El valor del alpha de Cronbach obtenido fue de .81 .

Todos los índices de fiabilidad obtenidos fueron $>.70$, superando los valores recomendados por Nunnally \& Bernstein (1994).

\section{Procedimiento}

Primeramente se obtuvo el permiso del Comité de Ética de la universidad de los investigadores. Posteriormente, se contactó con los diferentes centros educativos que participaron en la investigación para pedir su colaboración. Tras ello, se obtuvo el consentimiento informado de padres y madres de los participantes. Finalmente, el investigador principal administró los cuestionarios a todos los estudiantes durante la clase de educación física o tutoría. Se animó a los participantes a que contestaran lo más verazmente posible, informándoles de que sus respuestas eran totalmente anónimas y que en ningún caso afectaría a la calificación de la asignatura. La duración aproximada para administrar los cuestionarios fue de 20 minutos.

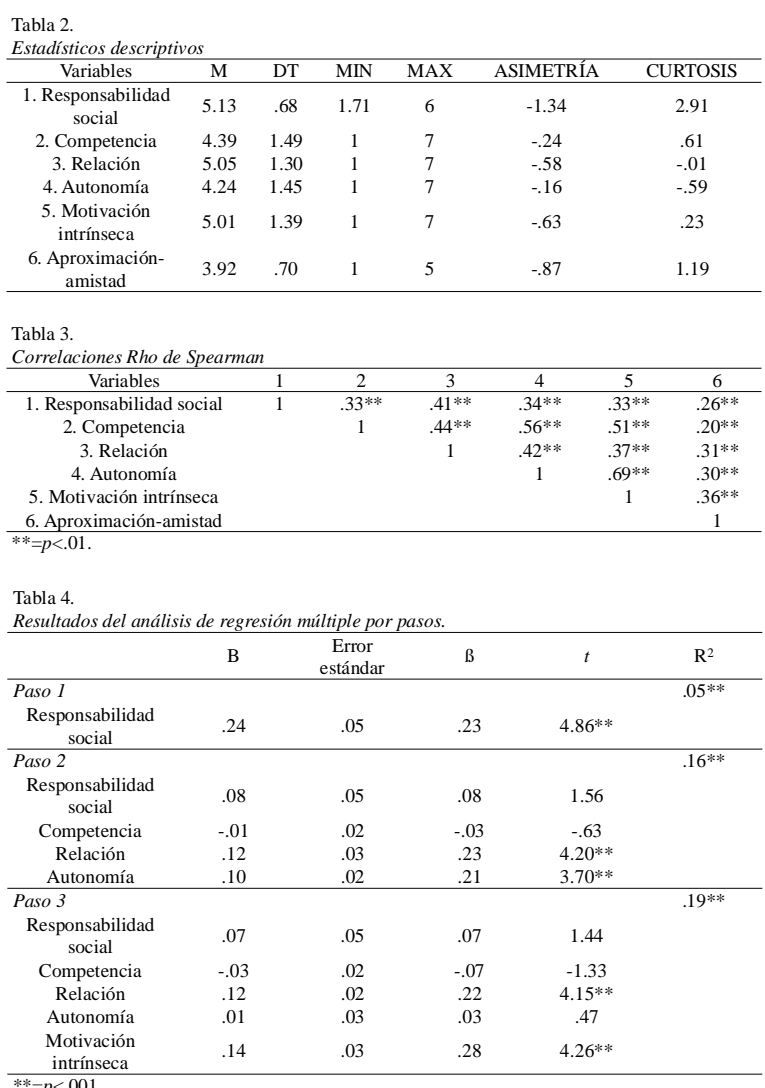

\section{Análisis de datos}

Los análisis estadísticos de este estudio fueron realizado a través del SPSS 22.0 (IBM, 2013). En primer lugar, se realizó la prueba de Kolmogorov-Smirnov para analizar la distribución de los datos. Se obtuvieron valores a $<.05$ en todas ellas. Esto significa que no se cumple el criterio de normalidad. A continuación se calcularon los estadísticos descriptivos (medias, desviaciones típicas, mínimos, máximos, asimetría y curtosis) y las correlaciones de Rho de Spearman. Posteriormente se realizó un análisis de regresión múltiple por pasos para conocer el grado de predicción de la responsabilidad social, las necesidades psicológicas básicas y la motivación intrínseca sobre la meta de aproximación-amistad.

\section{Resultados}

\section{Estadísticos descriptivos y correlaciones bivariadas}

En la Tabla 2 se presentan los estadísticos descriptivos de la responsabilidad social, las tres necesidades psicológicas básicas, la motivación intrínseca y la meta de aproximación-amistad, así como la asimetría y la curtosis de las variables. Se observa que el valor más alto es el de la variable responsabilidad social (M=5.13), mientras que el más bajo el de la aproximación-amistad (M=3.92). En la Tabla 3 se muestran los coeficientes de correlación Rho de Spearman. Se observan correlaciones significativas en todas las variables de estudio, siendo la más alta la que se produce entre la autonomía y la motivación intrínseca $(r=.69 ; p<.001)$, y la más baja la que se da entre la competencia y la aproximaciónamistad $(r=.20 ; p<.001)$.

\section{Análisis de regresión lineal múltiple por pasos}

En aras de predecir la aproximación-amistad de los participantes de la investigación se realizó un análisis de regresión múltiple por pasos (tres pasos). Por tanto, se introdujeron como variables predictoras/ independientes la responsabilidad social, las tres necesidades psicológicas básicas (relación, autonomía y competencia), y la motivación intrínseca, mientras que la variable independiente fue la aproximación-amistad. La introducción de las variables mediante este procedimiento se hizo con base a las aportaciones del modelo jerárquico de Vallerand (1997). De este modo, en el primer paso se introdujo el factor social (responsabilidad social), en el segundo se añadieron los mediadores psicológicos (las tres necesidades psicológicas básicas) y en el último de los pasos la regulación motivacional (motivación intrínseca).

En la Tabla 3 se pueden observar los resultados del análisis de regresión, el cual ha reportado tres modelos significativos. En el primero de los pasos en el que se incluyó como factor social la responsabilidad social, los resultados mostraron un coeficiente de regresión significativo, siendo por tanto una variable predictora de la aproximación-amistad ( $\beta=.23 ; p<.001)$. En este paso se explicó un $5 \%$ de la varianza. El segundo de los pasos en el que se introdujeron los mediadores psicológicos se explicó un $16 \%$ de la varianza. Las necesidades psicológicas de relación ( $\beta=.23 ; p<.001$ ) y autonomía ( $\beta=.21 ; p<.001)$ fueron las variables predictoras que mostraron coeficientes de regresión significativos. Finalmente, el tercer paso, en el que también se incluyó la motivación intrínseca como regulador motivacional, explicó un 19\% de la varianza. La relación ( $\beta=.22 ; p<.001)$ y la motivación intrínseca $(\beta=.28 ; p<.001)$ fueron las variables predictoras que presentaron coeficientes de regresión significativos.

\section{Discusión}

El objetivo principal de esta investigación fue analizar la capacidad de predicción de la responsabilidad social, las necesidades psicológicas básicas y la motivación intrínseca sobre la meta de aproximación-amistad. La hipótesis principal que se planteó fue que la responsabilidad social, las necesidades psicológicas básicas de competencia, relación y autonomía y la motivación intrínseca predecirían positiva y significativamente la meta de aproximación-amistad. Los resultados del análisis de regresión lineal han mostrado que la responsabilidad social, 
las necesidades psicológicas básicas de relación y autonomía y la motivación intrínseca predijeron de forma significativa la meta de aproximación-amistad. Asimismo, los análisis de correlaciones bivariadas reflejaron correlaciones positivas y significativas entre la responsabilidad social, las tres necesidades psicológicas básicas, la motivación intrínseca y la meta de aproximación-amistad.

Los resultados obtenidos confirman casi totalmente la hipótesis planteada, pues de todas las variables objeto de estudio tan solo la necesidad psicológica básica de competencia no apareció como predictora de la meta de aproximación-amistad. Apesar de que no hay estudios que hayan analizado la meta de aproximación-amistad como consecuencia final dentro del modelo Vallerand, otros estudios han estudiado la misma secuencia, pero con diferentes factores sociales y consecuencias. Méndez-Giménez, Fernández-Río \& Cecchini-Estrada (2012) analizaron un modelo multiteórico de metas de logro, metas de amistad y autodeterminación dentro de las clases de educación física. Los resultados revelaron que la meta de aproximación-maestría predecía de forma significativa y positiva las tres necesidades psicológicas básicas y las metas de aproximación-amistad. Asu vez, las necesidades psicológicas básicas predecían la motivación intrínseca y esta, de forma consecuente, el autoconcepto físico y la diversión. Las correlaciones bivariadas también reflejaron correlaciones significativas y positivas en estas variables. Estos resultados guardan estrecha relación con los que aquí se han obtenido, pues, igualmente, la aproximación-amistad, las tres necesidades psicológicas básicas y la motivación intrínseca correlacionaron entre ellas de forma positiva y significativa. Estos datos dan apoyo a estudios anteriores de similares características dentro del Modelo de Vallerand (Ntoumanis, 2001; Standage et al., 2003).

Por su parte, Moreno et al. (2012) estudiaron la capacidad de predicción de las metas sociales, las necesidades psicológicas básicas y la motivación intrínseca sobre el esfuerzo. En ese estudio, al igual que en el que aquí se presenta, se encontraron correlaciones significativas entre la responsabilidad, las tres necesidades psicológicas básicas, la motivación intrínseca y el esfuerzo. Sin embargo, a pesar de las similitudes correlacionales de ambos estudios, cabe destacar que en la secuencia propuesta por Moreno et al. (2012), la competencia predijo la consecuencia final (el esfuerzo), mientras que en esta investigación fue la competencia la única variable que no predijo la consecuencia final (la meta de aproximación-amistad). Esto se pudo deber a que el esfuerzo y la competencia tienen una orientación más personal que social, como también se puede constatar en el trabajo de Cecchini, González-Mesa, Méndez-Giménez \& Fernández-Río (2008), en el que la meta de responsabilidad y la de relación no predijeron el esfuerzo, pero sí lo hizo la aproximación al rendimiento; otra variable que guarda estrecha relación con la necesidad de competencia, como se puede constatar en el trabajo de García-Romero (2015). Este aspecto se puede observar en el presente trabajo, puesto que no solo la competencia no predijo la meta de aproximación-amistad, sino que además fueron las dos variables que menos índice de correlación obtuvieron de todas las que fueron objeto de análisis. Sin embargo, diversos estudios han señalado la necesidad de competencia como una de las variables de mayor impacto sobre la motivación intrínseca (Standage et al., 2003). En el presente trabajo se ha podido constatar tal afirmación pues la correlación entre estas dos variables fue de las más altas. Esto indica que es necesario que en las clases de educación física se cree un clima donde se potencie la competencia percibida del alumnado, en aras de fomentar la motivación interna y, de forma consecuente, variables como la satisfacción con la vida (Méndez-Giménez, Cecchini-Estrada, Fernández-Río, Méndez-Alonso \& Prieto-Saborit, 2017) o la autoestima y la diversión (Moreno \& Vera, 2011), entre otras.

Asimismo, es destacable la importancia que se ha observado en la necesidad psicológica básica de autonomía, no solo como predictora de la aproximación-amistad, sino especialmente por la elevada correlación que guardó con la motivación intrínseca. Diversos estudios han constatado la importancia de este mediador para favorecer la motivación intrínseca (Cox \& Williams, 2008; Ntoumanis, 2001, 2005; Standage et al., 2003), que a su vez predice variables de gran relevancia como la diversión y el aburrimiento de forma positiva y negativa, respectivamente (García-González et al., 2015). Teniendo esto en consideración, parece ser de gran relevancia que se generen estrategias por el docente para propiciar climas donde el alumnado tenga el sentimiento de poder tener cierto control sobre las actividades que realiza. Esto implica emplear enfoques de enseñanza centrados en el alumnado y en sus intereses personales. De esta forma se puede conseguir un aumento en la motivación intrínseca que da como resultado un incremento en la meta de aproximación-amistad. En este estudio, esta regulación motivacional predijo dicha meta y se correlacionó con ella de forma significativa. De igual manera, el soporte a la autonomía puede traer importantes beneficios, pues esta necesidad se correlaciona negativamente con variables a eliminar dentro de las clases de educación física como es la oposición desafiante (Abós et al., 2016).

Las aplicaciones prácticas que se derivan de este estudio se pueden vincular hacia el empleo de modelos pedagógicos como la Educación Deportiva (MED Siedentop, Hastie \& Van Der Mars, 2011), el Aprendizaje Cooperativo (Johnson, Johnson \& Holubec, 2013; FernándezRío, 2017), el MRPS (Hellison, 2011) o las estrategias motivacionales del TARGET (Ames, 1992), que fomentan de forma significativa la responsabilidad personal y social (Caballero, 2015; Caballero, Delgado \& Escartí, 2013), la motivación intrínseca (Fernández-Río, Sanz, Fernández-Cando \& Santos, 2016; Wallhead, Garn \& Vidoni, 2014) y las tres necesidades psicológicas básicas (Cuevas, García-López \& Contreras, 2015; Menéndez \& Fernández-Río, en prensa). Así pues, creando contextos educativos mediante la utilización de estos enfoques se pueden llegar a conseguir las condiciones necesarias en las clases de educación física para fomentar la responsabilidad social, las necesidades psicológicas de competencia, relación y autonomía y la motivación intrínseca del alumnado. Esto puede conducir a que el alumnado perciba un clima en el que, gracias a la potenciación de estas variables, tenga la oportunidad de fomentar los necesarios vínculos de amistad, especialmente entre alumnos que no suelen tener relaciones asiduas durante las clases. Por lo tanto, el empleo de modelos pedagógicos se convierte en un aspecto fundamental dentro de la práctica docente en aras de estimular variables de gran calado dentro del contexto de la educación física, como las metas de aproximación-amistad.

Las limitaciones principales de esta investigación son tres: (1) tan solo tres centros de similares características socioeconómicas participaron en la investigación. Por tanto, futuras investigaciones deberán llevarse a cabo en centros de diversa procedencia socioeconómica para obtener unos resultados con mayor validez, (2) el tipo de muestreo ha sido de carácter intencional por accesibilidad. Próximos trabajos que aborden este tema deberían realizarse empleando a capo muestreos con mayor validez metodológica, como son los muestreos aleatorios, por estratos y/o conglomerados, y (3) el tipo de diseño metodológico, de carácter transversal y correlacional, impide cualquier tipo de explicaciones de índole causal. Estudios longitudinales y diseños experimentales y/o cuasi-experimentales deberían llevarse a cabo para comprobar la secuencia planteada en este estudio.

\section{Conclusiones}

Esta investigación ha reflejado la importancia que tiene el desarrollo de la responsabilidad social en contextos de educación física para desarrollar las necesidades psicológicas básicas de relación y autonomía y la motivación intrínseca de los estudiantes y promover las metas de aproximación-amistad en estos. Los resultados del presente estudio sugieren la importancia que tiene que el profesorado desarrolle contextos educativos que fomenten el desarrollo de estas variables a través de planteamientos basados en modelos pedagógicos (especialmente aquellos que fomentan las relaciones sociales como el MED, el MRPS o el MAC) u otro tipo de estrategias motivacionales como el TARGET.

Asimismo, la novedad de la inclusión de la metas de amistas (aproximación) como variable dentro del modelo jerárquico de Vallerand es un aspecto novedoso que puede abrir vías y opciones para que otros investigadores analicen el estudio de la motivación desde un punto de 
vista social en las clases de educación física. En ese sentido, la significativa conexión que se ha establecido entre la meta de aproximaciónamistad y las variables más sociales de la presente investigación (responsabilidad social y relación) son un importante punto de apoyo para analizar el aspecto social en el marco de la motivación.

\section{Referencias}

Abós, A., Sevil, J., Sanz, M., Aibar, A., \& García-González, L. (2016). El soporte de autonomía en Educación Física como medio de prevención de la oposición desafiante del alumnado. RICYDE. Revista internacional de ciencias del deporte, 43(12), 65-78. doi: 10.5232/ ricyde2016.04304

Allen, J. B. (2003). Social motivation in youth sport. Journal of Sport \& Exercise Psychology, 25(4), 551-567. doi: 0.1123/jsep.25.4.551

Ames, C. (1992). Achievement goals, motivational climate, and motivational processes. En G. C. Roberts (Ed.), Motivation in sport and exercise (pp. 161-176). Champaign, IL: Human Kinetics.

Bagozzi, R. P., \& Yi, Y.(1988). On the evaluation of structural equation models. Journal of the Academy of Marketing Science, 16(2), 7494.

Bisquerra, R. (coord.) (2012). Metodología de la investigación educativa. Madrid: La Muralla.

Caballero, P. (2015). Diseño, implementación y evaluación de un programa de actividades en la naturaleza para promover la responsabilidad personal y social en alumnos de formación profesional. Cuadernos de Psicología del Deporte, 15(2), 179-194.

Caballero, P., Delgado, M.A., \& Escartí,A. (2013). Analysis of Teaching Personal and Social Responsibility model-based programmes applied in USA and Spain. Journal of Human Sport and Exercise, 8(2), 427-441. Doi: 10.4100/jhse.2012.82.10

Cantú-Berrueto, A., Castillo, I., López-Walle, J., Tristán, J., \& Balaguer, I. (2016). Estilo interpersonal del entrenador, necesidades psicológicas básicas y motivación: un estudio en futbolistas universitarios mexicanos. Revista Iberoamericana de Psicología del Ejercicio yel Deporte, 11(2), 263-270.

Cecchini, J.A. González-Mesa, C., Méndez-Giménez,A. \& FernándezRío, J. (2011). Achievement goals, social goals, and motivational regulations in physical education settings. Psicothema, 23(1), 5157.

Cecchini, J. A., González-Mesa, C., Méndez-Giménez,A., FernándezRío, J., Contreras, O., \& Romero, S. (2008). Metas sociales y de logro, persistencia-esfuerzo e intenciones de práctica deportiva en el alumnado de educación física. Psicothema, 20(2), 260-265.

Cox,A., \& Williams, L. (2008). The roles of perceived teacher support, motivational climate, and psychological need satisfaction in students' physical education motivation. Journal of Sport y Exercise Psychology, 30(2), 222-239.

Cuevas, R., García-López, L. M., \& Contreras, O. (2015). Influencia del modelo de Educación Deportiva en las necesidades psicológicas básicas. Cuadernos de Psicología del Deporte, 15(2), 155-162

Deci, E. L., \& Ryan, R. M. (1985). Intrinsic motivation and selfdetermination in human behaviour. New York: Plenum Press.

Deci, E. L., \& Ryan, R. M. (2002). Overview of self-determination theory: An organismic dialectical perspective. En E. L. Deci \& R. M. Ryan (Eds.), Handbook of self-determination research (pp, 336). University of Rochester Press.

Elliot, A. J., \& McGregor, H. A. (2001). A 2x2 achievement goal framework. Journal of Personality and Social Psychology, 80(3), 501-519. doi: 10.1037/0022-3514.80.3.501

Elliot, A. J., Gable, S. L., \& Mapes, R. R. (2006). Approach and avoidance motivation in the social domain. Personality and Social Psychology Bulletin, 32(3), 378-391. doi: 10.1177/ 0146167205282153

Escartí, A., Gutiérrez, M., \& Pascual, C. (2011). Propiedades psicométricas de la versión española del cuestionario de responsabilidad personal y social en contextos de educación física. Revista de Psicología del Deporte, 20(1), 119-130.

Fernández-Río, J. (2017). El Ciclo del Aprendizaje Cooperativo: una guía para implementar de manera efectiva el aprendizaje cooperativo en educación física. Retos. Nuevas Tendencias en Educación Física, 32.

Fernández-Río, J., Cecchini-Estrada, J. A., \& Méndez-Giménez, A. (2014). A cluster analysis on students' perceived motivational climate. Implications on psycho-social variables. The Spanish Journal of Psychology, 17. doi: 10.1017/sjp.2014.21

Fernández-Río, J., Méndez-Giménez, A., Cecchini-Estrada, J. A., \& González de Mesa, C. (2012). La influencia de las metas de logro y las metas sociales sobre el fair play de estudiantes de Educación Física de Secundaria. Revista de Psicodidáctica, 17(1), 73-91

Fernández-Río, J., Sanz, N., Fernández-Cando, J., \& Santos, L. (2016). Impact of a sustained Cooperative Learning intervention on student Motivation. Physical Education and Sport Pedagogy. doi: 10.1080/ 17408989.2015.1123238

Franco, E., Coterón, J., Gómez, V., Brito, J., \& Martínez, H. (2017). Influencia de la motivación y del flow disposicional sobre la intención de realizar actividad físico-deportiva en adolescentes de cuatro países. Retos. Nuevas Tendencias en Educación Física, 31, 46-51.

García-González, L., Aibar, A., Sevil, J.,Almolda, F. J., \& Clemente, J. A. (2015). Soporte de autonomía en Educación Física: evidencias para mejorar el proceso de enseñanza. Cultura_ciencia_deporte, 10(29), 103-11. doi: 10.12800/ccd.v10i29.547

García-Romero, C. (2015). Relación entre las metas de logro 3x2 y la competenciapercibida en los estudiantes de educación física. Sportis. Revista Técnico-Científica del Deporte Escolar, Educación Físicay Motricidad, 1(3), 293-310.

González-Cutre, D., Sicilia, A., \& Moreno, J. A. (2008). Modelo cognitivo-social de la motivación de logro en Educación física. Psicothema, 20(4), 642-651.

Goudas, M., Biddle, S. J. H., \& Fox, K. (1994). Perceived locus of causality, goal orientations and perceived competence in school physical education classes. British Journal of Educational Psychology, 64(3), 453-463. Doi: 10.1111/j.20448279.1994.tb01116.x

Granero-Gallegos, A., Baena-Extremera, A., Sánchez-Fuentes, J.A., \& Martínez-Molina, M. (2014). Perfiles motivacionales de apoyo a la autonomía, autodeterminación, satisfacción, importancia de la educación física e intención de práctica en tiempo libre. Cuadernos de Psicología del Deporte, 14(2), 59-70.

Hellison, D. (2011). Teaching responsibility through physical activity (3th Ed.). Champaign, IL: Human Kinetics.

Hemphill, M. A., Templin, T. J., \& Wright, P. M. (2013). Implementation and outcomes of a responsibility-based continuing professional development protocol in physical education. Sport, Education, and Society, 20(3), 398-419 doi: 10.1080/ 13573322.2012.761966

IBM Corp. Released (2013). IBM SPSS Statistics for Windows, Version 22.0. Armonk, NY: IBM Corp.

Jiménez, M. G., Martínez P., Miró, E., \& Sánchez A. I. (2008). Bienestar psicológico y hábitos saludables: ¿están asociados a la práctica de ejercicio físico? International Journal of Clinical and Health Psychology, 8(1), 185-202.

Johnson, D. W., Johnson, R. T., \& Holubec, E. J. (2013). Cooperation in the Classroom (9th ed.). Edina, MN: Interaction Book Company.

Li, W., Wright, P. M., Rukavina, P.B., \& Pickering, M. (2008). Measuring students' perceptions of personal and social responsibility and the relationship to intrinsic motivation in urban physical education. Journal of Teaching in Physical Education, 27(2), 167-178.

Méndez-Giménez,A., Fernández-Río, J., Cecchini, J.A. (2012). Análisis de un modelo multiteórico de metas de logro, metas de amistad y autodeterminación en educación física. Estudios de Psicología, 33(3), 325-336.

Méndez-Giménez, A., Cecchini-Estrada, J. A., \& Fernández-Río, J. (2013a). Climas motivacionales, necesidades, motivación y resul- 
tados en Educación Física. Aula Abierta, 41(1), 63-72.

Méndez-Giménez, A., Cecchini-Estrada, J. A., \& Fernández-Río, J. (2013b). Papel importante del alumnado, necesidades psicológicas básicas, regulaciones motivacionales y autoconcepto físico en educación física. Cuadernos de Psicología del Deporte, 13(1), 71-82.

Méndez-Giménez, A., Cecchini-Estrada, J.A., \& Fernández-Río, J. (en prensa). El modelo de Vallerand en adolescentes asturianos: implementación y extensión. Revista Internacional de Medicina y Ciencias de la Actividad Física y el Deporte.

Méndez-Giménez, A., Fernández-Río, J., \& Cecchini-Estrada, J. A. (2014). Validación de la versión en española del cuestionario de metas de amistad en educación física. Universitas Psychologica, 13(1), 227-237. doi: 10.11144/Javeriana.UPSY13-1.vvec

Méndez-Giménez, A., Cecchini-Estrada, J. A., Fernández-Río, J., Méndez-Alonso, D., \& Prieto-Saborit, J. A. (2017). Metas de logro 3x2, motivación autodeterminada y satisfacción con la vida en Educación Secundaria. Revista de Psicodidáctica.

Menéndez, J. I., \& Fernández-Río, J. (en prensa). Versión española de la escala de necesidades psicológicas básicas en educación física. Revista Internacional de Medicina y Ciencias de la Actividad Física yel Deporte.

Menéndez, J. I., \& Fernández-Río, J. (2016a). Violencia, responsabilidad, amistad y necesidades psicológicas básicas: efectos de un programa de Educación Deportiva y Responsabilidad Personal y Social. Revista de Psicodidáctica, 21(2), 245-260. Doi: 10.1387/ RevPsicodidact.15269

Menéndez, J. I., \& Fernández-Río, J. (2016b). Hibridación de los modelos de Educación Deportiva y Responsabilidad Personal y Social: una experiencia a través de un programa de kickboxing educativo. Retos. Nuevas Tendencias en Educación Física, Deporte y Recreación, 30, 150-158.

Montero, I., \& León, O. G. (2005). Sistema de clasificación del método en los informes de investigación en Psicología. International Journal of Clinical and Health Psychology, 5(1), 115-127.

Monzonís, N., \& Capllonch, M. (2014). La educación física en la consecución de la competencia social y ciudadana. Retos. Nuevas Tendencias en Educación Física, Deportes y Recreación, 25, 180185.

Moreno, J. A., \& Vera, J. A. (2011). Modelo causal de la satisfacción con la vida en adolescentes de la educación física. Revista de Psicodidáctica, 16(2), 367-380.

Moreno, J. A., Águila, C., \& Borges, F. (2011). La socialización en la práctica físico-deportiva de carácter recreativo: predictores de los motivos sociales. Apunts. Educación Física y Deportes, 113(1), 7682.

Moreno, J. A., González-Cutre, D., y Chillón, M. (2009). Preliminary validation in Spanish of a scale designed to measure motivation in physical education classes: the Perceived Locus of Causality (PLOC) Scale. The Spanish Journal of Psychology, 12(1), 327-337

Moreno, J. A., González-Cutre, D., Chillón, M., \& Parra, N. (2008). Adaptación a la educación física de la escala de las necesidades psicológicas básicas en el ejercicio. Revista Mexicana de Psicología, 25(2), 295-303.

Moreno, J. A., Cervelló, E., Montero, C., Vera, J. A., \& García, T. (2012). Metas sociales, necesidades psicológicas básicas y motivación intrínseca como predictores de la percepción del esfuerzo en las clases de educación física. Revista de Psicología del Deporte, 21(2), 215-221.

Navarro-Patón, R., Rodríguez, J. E., \& Eirin, R. (2016). Análisis de la satisfacción de las necesidades psicológicas básicas, motivación y disfrute en Educación Física en Primaria. Sportis. Revista TécnicoCientífica del Deporte Escolar, Educación Física y Motricidad, 3(2), 439-455. doi:10.17979/sportis.2016.2.3.1758

Nicholls, J. G. (1989). The competitive ethos and democratic education. Cambridge, MA: Harvard University Press.

Niemec, C. P., \& Ryan, R. M. (2009). Autonomy, competence, and relatedness in the classroom: Applying Self-Determination Theory to educational practice. Theory and Research in Education, 7(2), 133-144. doi: $10.1177 / 1477878509104318$

Ntoumanis, N. (2001). A self-determination approach to the understanding of motivation in physical education. British Journal of Educational Psychology, 71(2), 225-242.

Ntoumanis, N. (2005). A prospective study of participation in optional school physical education using a self-determination theory framework. Journal of Educational Psychology, 97(3), 444-453. doi: 10.1037/0022-0663.97.3.444

Nunnally, J. C., \& Bernstein, I. H. (1994). Psychometric Theory. Nueva York: McGraw-Hill.

Sánchez, D., Leo, F. M., Sánchez, P. A., Gómez, F. R., \& García, T. (2011). Teoría de la autodeterminación y comportamientos prosociales en jóvenes jugadores de fútbol. Apunts. Educación Física y Deportes, 113(1), 31-37.

Sánchez-Alcaraz, B. J., Gómez-Mármol, A., Valero, A., \& De la Cruz, E. (2013). Aplicación de un programa para la mejora de la responsabilidad personal y social en las clases de educación física. Motricidad. European Journal of Human Movement, 30, 121-129.

Sari, Ý., Ekici, S., Soyer, F., \& Eskiler, E. (2015). Does Self-confidence Link to Motivation? A Study in Field Hockey Athletes. Journal of Human Sports and Exercise, 10(1), 24-35.

Seligman, M. E. P., \& Csikszentmihalyi, M. (2000). Positive Psychology: An Introduction. American Psychologist, 55(1), 5-14.

Severinsen, G. (2014). Teaching personal and social responsibility to juniors through physical education. Asia-Pacific Journal of Health, Sport and Physical Education, 5(1), 83-100. doi: 10.1080/ 18377122.2014.867793

Sevil, J., Abós, A., Generelo, E., Aibar, A., \& García-González, L. (2016). Importancia de apoyo a las necesidades psicológicas básicas en la predisposición hacia diferentes contenidos en Educación Física. Retos. Nuevas Tendencias en Educación Física, Deportes y Recreación, 29, 3-8.

Sevil, J., Abós, A., Julián, J. A., Murillo, B., y García-González, L. (2015). Género y motivación situacional en Educación Física: claves para el desarrollo de estrategias de intervención. RYCIDE. Revista Internacional de Ciencias del Deporte, 11(41), 281-296. doi: 10.5232/ricyde2015.04106

Siedentop, D., Hastie, P. A., \& Van Der Mars, H. (2011). Complete Guide to SportEducation (2nded.), Champaign, IL, Human Kinetics.

Standage M., \& Gillison, F. (2007). Students’ motivational responses toward school physical education and their relationship to general self-esteem and health-related quality of life. Psychology of Sport and Exercise, 8(5),704-721.

Standage, M., Duda, J.L., \& Ntoumanis, N.(2003). Amodel of contextual motivation in physical education: Using constructs from selfdetermination and achievement goal theories to predict physical activity intentions. Journal of Educational Psychology, 95(1), 97110. doi: 10.1037/0022-0663.95.1.97

Vallerand, R. J. (1997). Toward a Hierarchical Model of Intrinsic and Extrinsic Motivation. En M. P. Zanna (Ed.), Advances in Experimental Social Psychology (vol. 29, pp. 271-360). Nueva York: Academic Press.

Vlachopoulos, S. P., \& Michailidou, S. (2006). Development and initial validation of a measure of autonomy, competence, and relatedness in exercise: The Basic Psychological Needs in Exercise Scale. Measurement in Physical Education and Exercise Science, 10, 179 201. doi: $10.1207 /$ s15327841mpee1003_4

Vlachopoulos, S. P., Katartzi, E. S., \& Kontou, M. G. (2011). The basic psychological needs in physical education. Journal of Teaching in Physical Education, 30(3), 263-280. doi: 10.1123/itpe.30.3.263

Wallhead, T. L., Garn, A. C., \& Vidoni, C. (2014). Effect of a Sport Education Program on Motivation for Physical Education and Leisure-Time Physical Activity. Research Quarterly for Exercise and Sport, 84(5), 478-487. doi: 10.1080/02701367.2014.961051 